\title{
On the accuracy of capillary flow porometry for fibrous filter media
}

\author{
H.E. Kolb*, R. Schmitt, A. Dittler, G. Kasper \\ Institut für Mechan. Verfahrenstechnik und Mechanik, Karlsruhe Institute of Technology, Germany
}

Keywords:

Fibrous media

Characterization

Porometry

Volatility

Wetting liquid

\begin{abstract}
A B S T R A C T
The application of capillary flow porometry by gas-liquid displacement to the measurement of the pore size distribution in identical glass microfiber filter media can lead to surprisingly divergent results. The causes for these differences as well as the factors that influence the over-all reliability of data obtained by this widely used technique are investigated. Among the key factors studied were the volatility and viscosity of four common wetting liquids, the scan rate (i.e. the holding time between increments of differential pressure $\Delta \mathrm{p}$ or volumetric flowrate $\dot{V}$ ), and the scan sequence (i.e. dry before wet, or wet before dry scan). Most measurements were made with a porometer designed in house, in order to have complete control over all aspects of operation. Data obtained with commercial porometers are also reported. For best comparability, all measurements were made with the same batch of standard glass microfiber media.

The largest error source by far was the volatility of fluorinated compounds commonly used as wetting liquids. While the vapor pressures of such compounds may be relatively low, their use in combination with a flow of air through the porous matrix can have an enormous effect on the evaporation rate during a scan. Neglecting this effect (which obviously depends on the scan rate) may ultimately result in an error of almost arbitrary magnitude in the pore size distribution. Silicone oil on the other hand has a negligible volatility and provides reliable results for a wide range of operating conditions. The liquid viscosity in the tested range of $5-100 \mathrm{~mm}^{2} / \mathrm{s}$ played a comparatively insignificant role. These and other factors of uncertainty are discussed on the basis of experimental data.
\end{abstract}

\section{Introduction}

Capillary flow porometry is a well established technique for mea suring pore size distributions in polymer membranes and fibrous media, with a useful range of typically about $150 \mu \mathrm{m}$ for the latter. Its oper ating principle, as suggested by Erbe [1], is based on saturating small samples of the media completely with a wetting liquid, and then pro gressively "discharging" pores with a second fluid by increasing the differential pressure $\Delta \mathrm{p}$ across the sample. From the attendant increase in volumetric flow rate (or flow velocity) through the sample one can then derive a cumulative pore size distribution. The displacing fluid can be either air, as in most commercial porometers, or another liquid [2].

Assuming the pores have an ideally circular cross section, the re lationship between the differential pressure $\Delta \mathrm{p}$ and the smallest empty pore $d_{\text {pore }}$ is given by classical capillary theory, as

$d_{\text {pore }}=4 \frac{\gamma \cos (\Theta)}{\Delta p}$

with corrections required for a non ideal pore morphology [3,4]. As suming further that the surface tension $\gamma$ and the wetting angle $\Theta$ are constant for the entire internal surface of the media, the pore size distribution can be calculated from the ratio of the respective volu metric air flow rates through the dry and the wet media, obtained at the same $\Delta \mathrm{p}$ :

$Q(\Delta p)=1-\frac{\dot{V}_{w e t}}{\dot{V}_{d r y}}$

$\mathrm{Q}(\Delta \mathrm{p})$ is the cumulative number distribution of (equivalent) pore dia meters $d_{\text {pore }}$ as given by Eq. (1). The largest pore diameter (i.e. at $\mathrm{Q}=1$ ) corresponds to the bubble point (as defined e.g. by ASTM F316 [5]) and is relatively easy to establish. On the other hand, the smallest pore diameter (at $\mathrm{Q}=0$ ) depends on the maximum $\Delta \mathrm{p}$ sustainable by the media and possibly other external factors. A method of extending the lower limit of detectable pores is discussed by Hernandez et al. [6]. This technique will not be used here, however.

Capillary flow porometry is quite attractive to characterize porous media for applications such as filtration [7 9], catalysis [10] or chro matography [11]. Being a flow based method, it is often preferable to purely geometric or tomographic techniques such as described by Lehmann et al. [12] or Hoferer et al. [13], and also less cumbersome. Porometers are thus available commercially, and ASTM F316 describes their use for "non fibrous membranes". Despite such widespread use,

\footnotetext{
* Corresponding author.

E-mail address: eckhard.kolb@kit.edu (H.E. Kolb).
} 
the scientific literature contains few critical analyses of the method's fundamental reliability. The ASTM guideline F316 is vague with regard to details of applying the method.

Yunoki et al. [14] investigated the influence of three wetting fluids (alcohol, Porofil ${ }^{\mathrm{TM}}$ and ethylene glycol) on the pore size distribution of fibrous media. The authors observed a tendency of the distribution to become narrower and shift towards larger pores, which they correlated with the viscosity of the fluid and attributed to kinetic effects during the blow out of wetting liquid. The same paper also shows the effect of scanning rate, as will be discussed later.

Dixon [15] also discusses the impact of a broad range of wetting fluids on capillary flow porometry as applied to filter media. This hard to access conference paper also describes a "scatter" among the wet curves for more volatile liquids such as alcohol, and a relative in dependence of the bubble point. On the other hand, a "reduced effect of volatility for smaller pores" was observed.

Due to our interest in obtaining reliable pore size data for glass fiber filter media, we have in the past conducted comparative tests with several commercial porometers. The results (presented in the next section of this paper) showed very good reproducibility when repeated on the same ma chine and with the same sample material, but varied considerably between porometers. The reasons for these variances were not immediately clear, but may have been due to a number of reasons, including differences in the properties of the wetting liquids recommended by the respective porometer manufacturers, in scanning times of $\Delta \mathrm{p}$ or $\dot{\mathrm{V}}$, in sample area, and perhaps also unknown details of the proprietary software routines to convert raw data into pore size distributions.

Consequently, we conducted a more thorough study on the relia bility and comparability of this important technique, focusing on the influence of wetting fluids and the way a porometer is operated. In order to make the influence of these parameters fully transparent and independent of any specific commercial device, the measurements re ported here are based on a laboratory prototype device designed spe cifically for that purpose. On the other hand, the experiments reported hereunder were limited to a single type of glass microfiber media, which can be considered representative of an entire class of such media.

\section{Preliminary comparison tests with commercial porometers}

The evaluation included three porometers, a CFP 1500 AFX (PMI Inc.), a $3 G z H$ (Quantachrome GmbH), and a PSM 165 (Topas GmbH). Of these, the PMI device is available in house, the other measurements are courtesy of the respective instrument manufacturers with samples of glass microfiber of the same media investigated later in this paper.

Sample results for one typical filter medium are shown in Fig. 1 to characterize the differences in resulting pore size distribution. Evidently, the differences are substantial in various aspects of the distribution. Values for the largest pore corresponding to the "bubble point" normally very easy and reliable to determine range from $16 \mu \mathrm{m}$ (Topas) to $20 \mu \mathrm{m}$ (PMI), with the value for Quantachrome (red curve) in between. The $d_{50}$ values agree for two of the three instruments (around $7 \mu \mathrm{m}$ ), while the third instrument gives a larger value of about $10 \mu \mathrm{m}$. Both the upper and the lower ranges of the respective distributions differ substantially with regard to shape and contribution to the distribution. Especially the red curve (labeled Quanta chrome) appears to be multimodal on the upper end, while the others are mono modal. In fact, the only thing all three porometers seem to agree on is the minimal pore size around $5 \mu \mathrm{m}$.

Data for other tested media show a similar spread of values, but are not presented here, because more data would add nothing further to the description of the problem. Despite the observed deviations between instruments, repeated measurements on the same device produced nearly identical pore size distributions. (To some extent, this eliminates differences in sample size from the list of candidate causes.)

In sum, the differences between the respective curves are substantial and far too big to make these data useful for inter comparisons of media, or the interpretation of filter behavior. Considering furthermore

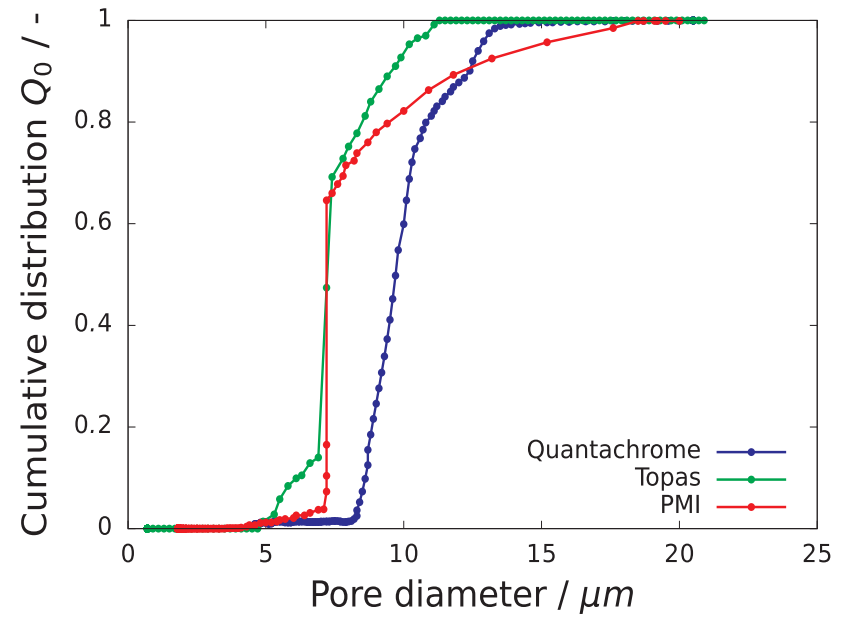

Fig. 1. Pore size distributions obtained by 3 different porometers for the identical type of glass fiber filter media. Working fluids used and scan procedures are those recommended/ implemented by the instrument manufacturers.

that the operation of a porometer involves only standard measurements of flow and pressure, these results are hard to explain. They cannot simply result from a translational shift due to some kind of calibration offset. Even though some deviations may be due to differences in in strument design and/or operation (e.g. sample area or scan time), other, major questions remain regarding the influence of the wetting fluids, the scan mode ( $\Delta \mathrm{p}$ scan vs. $\dot{V}$ scan), or the software.

\section{Experimental set-up and methods}

A porometer consists of only three essential components, an open face sample holder, a differential pressure gauge, and a device (such as a mass flow controller) to measure and control the airflow, as shown in Fig. 2. We therefore chose to build our own, in order to have all factors under our control.

The circular filter sample had an effective diameter of $14 \mathrm{~mm}$, which constituted a compromise between mechanical strength and a re presentative sample area with sufficient pores. Also, the area was large enough compared to the clamped fringes. The sample holder was checked for leak tightness.

For the sake of better comparison, all measurements reported hereunder were performed on the same type and batch of glass microfiber filter media, a standard commercial product (Hollingsworth \& Vose) with a mean fiber diameter of $1.6 \mu \mathrm{m}$, a porosity of $95 \%$, and a thickness of about $0.5 \mathrm{~mm}$. These media show spontaneous liquid wicking and may be considered fully wettable to all the liquids used in this work. The filter material was chosen because of its relatively good uniformity, which eliminates one potential error source from the measurements.

A flow of clean and dry compressed air was fed to the sample holder

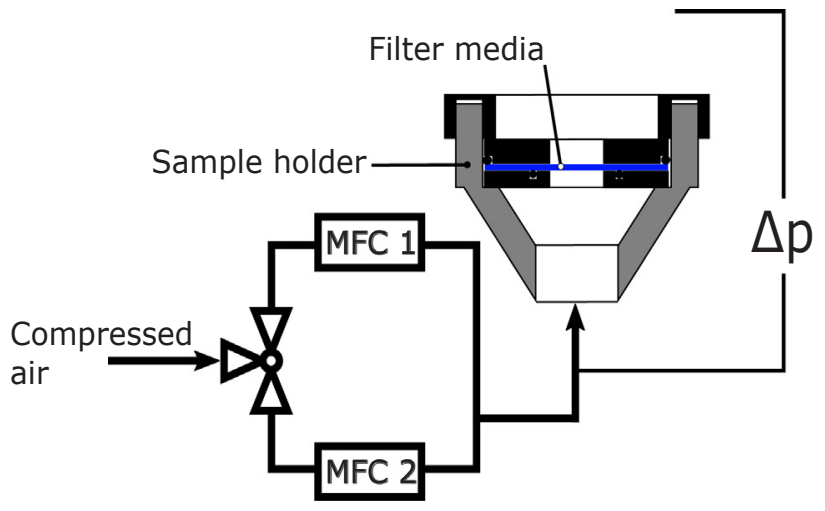

Fig. 2. Schematic diagram of the prototype porometer operating at differential pressures of 0-1000 mbar and volume flows of $0-200 \mathrm{~L} / \mathrm{min}$ (by one of two mass flow controllers, MFC). 


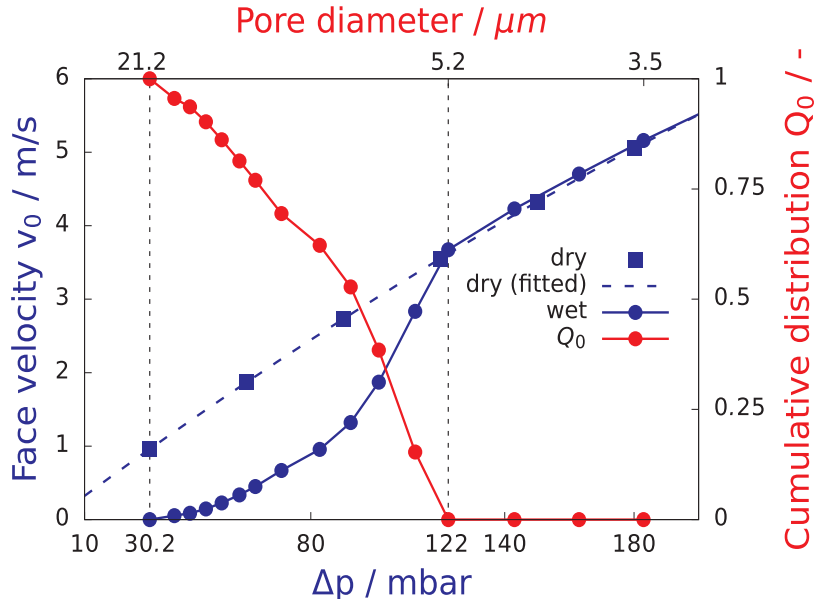

Fig. 3. "Wet" and "dry" curves (blue) of flow vs. $\Delta \mathrm{p}$. The "dry" curve is fitted with a secondorder polynomial for interpolation purposes (dashed line). The resulting pore size distribution (red) lies between 5.2 and $21.2 \mu \mathrm{m}$ for a scan time interval of $1 \mathrm{~min}$ in a pressure controlled run. The wetting liquid is Galwick ${ }^{\mathrm{TM}}$. (For interpretation of the references to color in this figure legend, the reader is referred to the web version of this article.)

via one of two mass flow controllers having different operating ranges (MKS 1179A for $04.7 \mathrm{~L} / \mathrm{min}$, MKS 1579A for $4.7200 \mathrm{~L} / \mathrm{min}$ ). A pressure transducer (Omega PD 23, 01000 mbar) measured the pres sure drop across the sample. Both airflow and pressure drop were re corded continuously by a computer.

The system could be operated either by setting $\Delta \mathrm{p}$ in predetermined increments and measuring the resulting flow rates, or vice versa. Both possibilities were realized as discussed later on. Experiments were al ways conducted in constant increments of scanning time, during each of which the controlling process variable was held constant while the dependent variable was recorded. These time increments were chosen

Table 1

Wetting liquids per recommendation of the respective instrument supplier with properties at STP conditions, if available.

\begin{tabular}{lllll}
\hline & Brand name & $\begin{array}{l}\text { Surface } \\
\text { tension } \gamma \\
\mathrm{mN} / \mathrm{m}\end{array}$ & $\begin{array}{l}\text { Kinematic } \\
\text { viscosity } \nu \\
\mathrm{mm}^{2} / \mathrm{s}\end{array}$ & $\begin{array}{l}\text { Vapor } \\
\text { pressure } \\
\text { Pa }\end{array}$ \\
\hline Fluorocarbons & $\begin{array}{l}\text { Galwick }^{\mathrm{TM}} \\
\text { Porofil }^{\mathrm{TM}}\end{array}$ & 15.9 & $\mathrm{NA}$ & $1-20$ \\
& Topor $^{\mathrm{TM}}$ & 16.0 & $\mathrm{NA}$ & NA \\
Silicone oils & Wacker $^{\mathrm{TM}}$ AK 5 & 16.0 & 2 & Approx. 400 \\
& Wacker $^{\mathrm{TM}}$ AK 10 & 20.0 & 5 & NA \\
& Wacker $^{\mathrm{TM}}$ AK 100 & 21.0 & 100 & NA \\
\hline
\end{tabular}

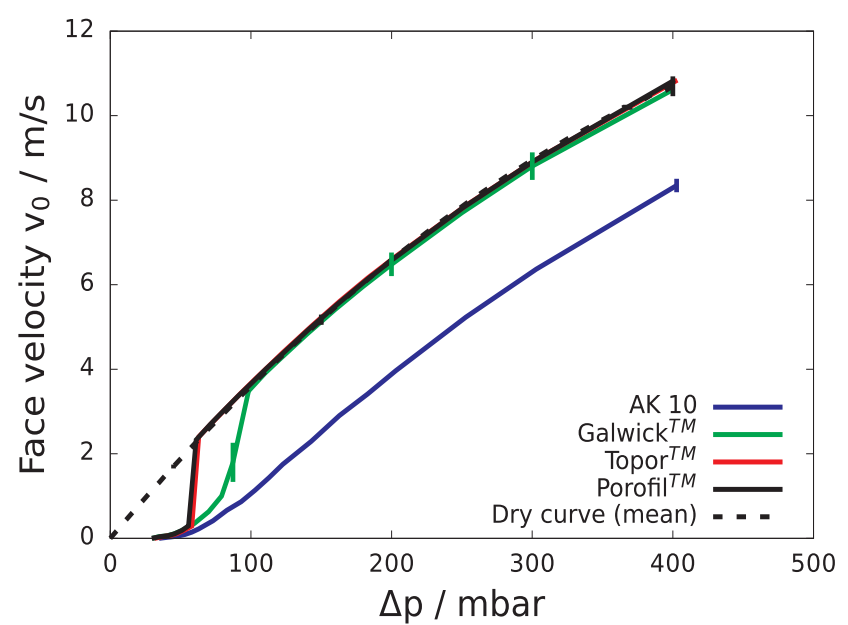

as $1,2,10,20,60$ or $120 \mathrm{~min}$.

Unless noted otherwise, each measurement sequence consisted of two separate runs (Fig. 3, bottom). First a "dry" curve of $\Delta \mathrm{p}$ vs. $\dot{V}$ was recorded. Immediately following the dry measurement, the sample was saturated completely with a constant amount $(200 \mu \mathrm{l})$ of one of the recommended wetting liquids (Table 1), without dismounting it from the sample holder. With this amount of liquid, the level of fluid above the sample is less than $1 \mathrm{~mm}$ and therefore negligible for the differential pressure drop. Finally, a "wet" measurement was performed by raising $\Delta \mathrm{p}$ across the sample at a rate of less than $1 \mathrm{mbar} / \mathrm{min}$ via the lower range MFC. The onset of bubble formation, i.e. the bubble point, was determined visually. Before starting, each sample was blown out with dry compressed air in order to remove any remaining humidity from its pores.

The pore size distribution was then calculated point by point ac cording to Eq. (2) from the respective airflow rates of the wet and dry curves. In order to obtain exactly corresponding values of the flow for a given $\Delta \mathrm{p}$, the dry curve was fitted by a 2 nd order polynomial. This then permitted interpolation to the same $\Delta \mathrm{p}$. For the subsequent graphs and discussion we have chosen to replace volumetric flow rates by the corresponding sample face velocity, because this is more general and eliminates the dependency on the sample diameter.

\section{Results \& discussion}

This section addresses and discusses the influence of various ex perimental factors and potential error sources on the porometry mea surement, starting with the most important one, namely the properties of the wetting fluid.

\subsection{Influence of the volatility of the wetting liquid}

Fig. 4 shows the wet and dry scans (left hand figure), as well as the resulting pore size distributions (right hand figure) for each of the four working liquids, including the non volatile silicone oil (Wacker ${ }^{\mathrm{TM}} \mathrm{AK}$ 10) and the more or less volatile fluorocarbons Galwick ${ }^{\mathrm{TM}}$, Topor ${ }^{\mathrm{TM}}$ and Porofil $^{\mathrm{TM}}$. Note that only the mean dry curve is shown (black dashed line going through the origin) because the individual ones overlap almost perfectly. The $\Delta \mathrm{p}$ controlled scan was stopped at $400 \mathrm{mbar}$.

The resulting pore size distributions in the right hand figure are each the average of three separate runs. Error bars indicating the re spective standard deviation have been inserted where feasible, because errors where mostly negligible on the scale of the graphs. Repeatability was generally very good, indicating that the filter material was indeed acceptably homogenous in pore structure and that the sample diameter was sufficient for a reliable measurement.

In Fig. 4 one notices that the onset of flow (at the bubble point)

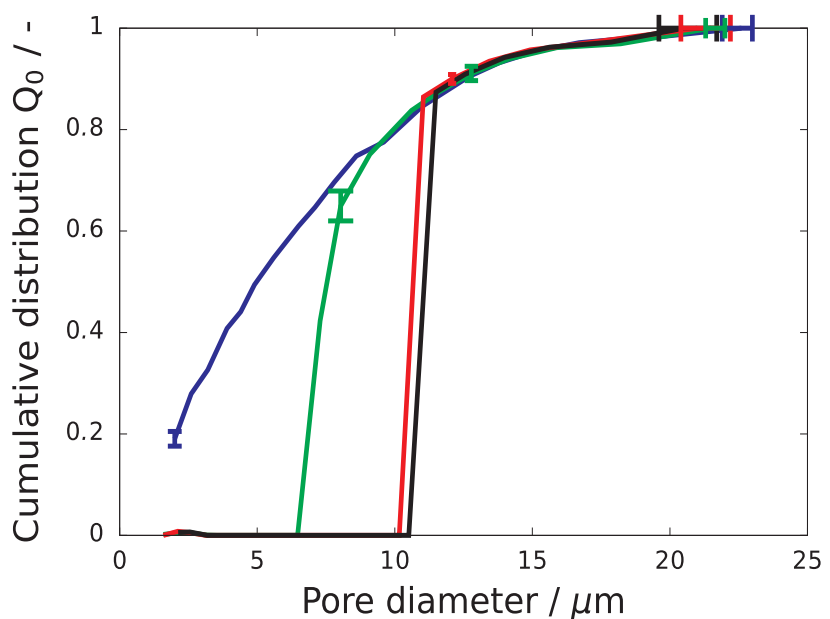

Fig. 4. Wet and dry curves (left) and resulting pore size distributions (right) for 4 different wetting fluids. Measurements were $\Delta$ p controlled with constant scanning time intervals of 2 min each. 


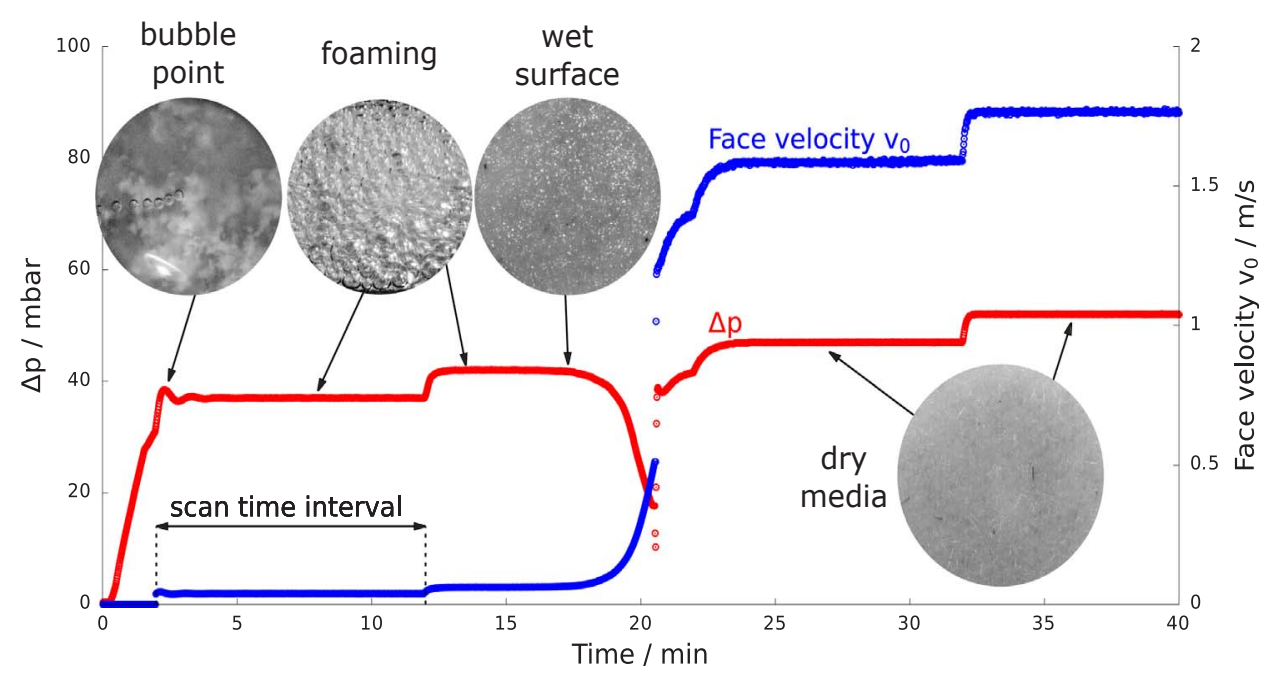

Fig. 5. Temporal evolution of $\Delta \mathrm{p}$ and face velocity during a $\Delta \mathrm{p}$-controlled scan. Insets show a top view of the sample surfaces at different stages. Data are for Porofil $^{\mathrm{TM}}$, the most volatile liquid.

occurs relatively consistently at $30.3 \pm 1.5$ mbar for Galwick ${ }^{\mathrm{TM}}$, Por ofil $^{\mathrm{TM}}$ and Topor ${ }^{\mathrm{TM}}$, and at $35.7 \mathrm{mbar}$ for silicone oil, corresponding to largest pores of 21.1 and $22.4 \mu \mathrm{m}$, respectively. The differences be tween the "wet" curves become more striking however toward smaller pores: while the silicone oil based curve (blue) continues smoothly toward the predetermined endpoint at $400 \mathrm{mbar}$, the other three curves break off abruptly and prematurely and then join their dry curves. Note also, that the pore size distribution for silicone oil ends around $1 \mu \mathrm{m}$, with about $25 \%$ of the pores not yet opened at the maximum pressure. (The influence of maximum $\Delta \mathrm{p}$ will be discussed later.)

Interpreted on face value, the above results simply mean that the per centage fine pores in the media depends on the liquid chosen. Another, more coherent explanation for the observed differences is the likely vola tility of three of the working fluids. Volatility effects become apparent when observing the liquid film on the exposed surface of the media during a $\Delta p$ scan, as shown in Fig. 5 for the case of Porofil ${ }^{\mathrm{TM}}$. The inserted photos show the liquid film at different stages of the scan, while the red and blue curves shown the raw data for $\Delta \mathrm{p}$ and flow rate vs. scan time.

At the beginning of a wet scan, the media surface was covered by a thin film of liquid. As $\Delta \mathrm{p}$ approaches the bubble point, several thin threads of rising bubbles became visible in the thin liquid layer. A further small in crease in flow induced foaming, at first weakly, then more vigorously. With increasing intensity, the foam soon disappeared (due to wall flow and bursting bubbles), leaving behind a humid media surface. A further small increase in $\Delta \mathrm{p}$ and flow caused the remaining liquid in the pores to eva porate completely, leaving behind an apparently dry surface (around $\mathrm{t}=21 \mathrm{~min}$ ). This point in time coincided with the steep increase in flow through the media seen in Fig. 4, and the convergence of the wet and the dry curves. The rate of disappearance of the foam was highest for Porofil ${ }^{\mathrm{TM}}$ (around $40 \mathrm{mbar}$, as seen in Fig. 5) and lowest for the silicone oil, where the foam remained up to about 100 mbar; Galwick $^{\mathrm{TM}}$ lay in between at 70 mbar. The abrupt drop in wet $\Delta \mathrm{p}$ down to the level of the dry $\Delta \mathrm{p}$ observed for both Porofil $^{\mathrm{TM}}$ and Galwick ${ }^{\mathrm{TM}}$, and the resulting sharp increase in air flow through the media are due to the complete evaporation of the liquid at that point in time. The rate of evaporation obviously depends on the volatility, which explains why this phenomenon happens more quickly for Porofil ${ }^{\mathrm{TM}}$ but was never observed for silicone oils; and it is dramatically accelerated by the increasing air flow, which explains the sharpness of the transition, in spite of the rather low vapor pressures of these liquids.

The sooner this evaporation (transition of the wet surface to dry media) took place during a scan, the narrower the resulting pore size distribution became. In case of Porofil ${ }^{\mathrm{TM}}$, this resulted in a cut off around $9 \mu \mathrm{m}$, for Galwick ${ }^{\mathrm{TM}}$ around $7 \mu \mathrm{m}$, while for silicone oil one obtains pore size information down to $1 \mu \mathrm{m}$, where the scan ended at $\Delta \mathrm{p}_{\max }$. The discharging of silicone oil filled pores prior to $\Delta \mathrm{p}_{\max }$ is also visible from a transition of the wetted media: the glossy surface, im mediately after the disappearance of foam, is followed by a decreasing humidity without returning to the completely dry media surface as for Galwick $^{\mathrm{TM}}$ and Porofil ${ }^{\mathrm{TM}}$.

As a further consequence of the premature and complete convergence of wet and dry curves in the cases of a volatile liquid, the calculated pore size distribution appears to go down to $Q=0$. One is thus lead to believe erroneously that the complete pore size distribution has been obtained. In fact, some 18 to $20 \%$ of pores have been missed at $\Delta \mathrm{p}_{\max }=400 \mathrm{mbar}$, as indicated by the blue curve for silicone oil in Fig. 4 .

More direct evidence for the volatility of the various wetting liquids was collected in a separate experiment, where the weight loss by eva poration of an initially saturated filter sample was measured over a time period of a few hours. To emulate the situation in a porometer more closely, a constant airflow through the filter sample was maintained during the test. The airflow of $5 \mathrm{~cm} / \mathrm{s}$ resulted in an initial $\Delta \mathrm{p}$ of approximately $50 \mathrm{mbar}$, which enables flow only in pores $>15 \mu \mathrm{m}$.

Fig. 6 shows the resulting change in saturation (i.e. the percentage of void space filled with liquid) with time due to evaporation of liquid from initially fully saturated samples. While the change over several hours was negligible for AK 10 as expected, the other two fluorocarbons behaved according to their volatility, and in accordance with the ob servations described in Fig. 4. Even though the fluorocarbons may have a relatively low vapor pressure at room temperature, the airflow through the samples accelerates the kinetics of the evaporation greatly.

Based on the gravimetric measurements of Fig. 6, the evaporation rates of the three liquids are calculated and compared with corre sponding characteristic pore sizes in Table 2 (extracted from Fig. 4).

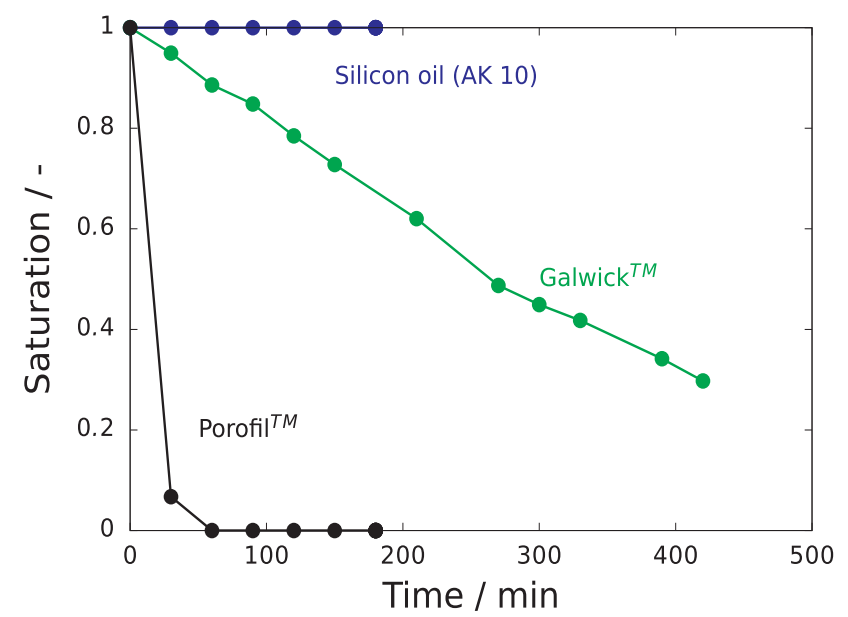

Fig. 6. Decrease in liquid saturation of filter samples with time due to evaporation. Samples were saturated fully at $\mathrm{t}=0$ with one of the 4 wetting liquids. A flow was maintained through the sample at $5 \mathrm{~cm} / \mathrm{s}$ face velocity at an initial $\Delta \mathrm{p}$ of $45 \mathrm{mbar}$ corresponding to pore sizes $>15 \mu \mathrm{m}$. 
Table 2

Evaporation rates and characteristic pore sizes measured with different wetting liquids. The error of largest/mean pore sizes is based on the standard deviation of 3 measurements.

\begin{tabular}{llll}
\hline Wetting liquid & $\begin{array}{l}\text { Evaporation rate } \\
\left(@ \mathrm{v}_{0}=0.05 \mathrm{~m} / \mathrm{s}\right) \\
\mathrm{g} /\left(\mathrm{m}^{2} \mathrm{~s}\right)\end{array}$ & $\begin{array}{l}\text { Largest pore } \\
\mu \mathrm{m}\end{array}$ & $\begin{array}{l}\text { Mean pore } \\
\mu \mathrm{m}\end{array}$ \\
\hline Wacker $^{\mathrm{TM}}$ AK 10 & $<0.0000$ & $22.6 \pm 0.62$ & $5.09 \pm 0.11$ \\
Galwick $^{\mathrm{TM}}$ & 0.0138 & $21.51 \pm 0.40$ & $7.59 \pm 0.08$ \\
Porofil & & $20.48 \pm 1.09$ & $11.12 \pm 0.07$ \\
\hline
\end{tabular}

The diameter of the largest pores are located within their error bars, while the mean pore sizes $(Q=0.5)$ move towards the largest pores for an increase in the evaporation rate. The slight decrease of the largest pores with the evaporation rate might be an indicator for a better wettability. This minor advantage, however, puts the reliability of the remaining pore size distribution at risk, as the systematic error due to volatility of the wetting liquid increases.

\subsection{Influence of the viscosity of the wetting liquid}

As mentioned earlier, Yunoki et al. [14] had already noticed dis crepancies between pore size distributions obtained by a porometer when using different wetting fluids, which were attributed to differ ences in viscosity. We therefore made measurements under identical conditions with three silicone oils having kinematic viscosities between

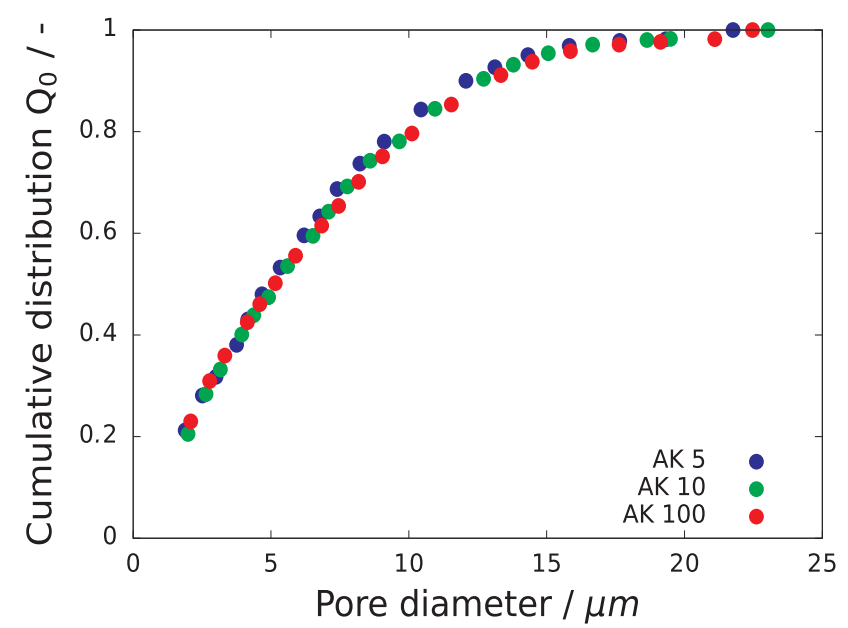

Fig. 7. Pore size distributions obtained with different viscosity grades of silicone oil (scan time intervals $2 \mathrm{~min}$ ).

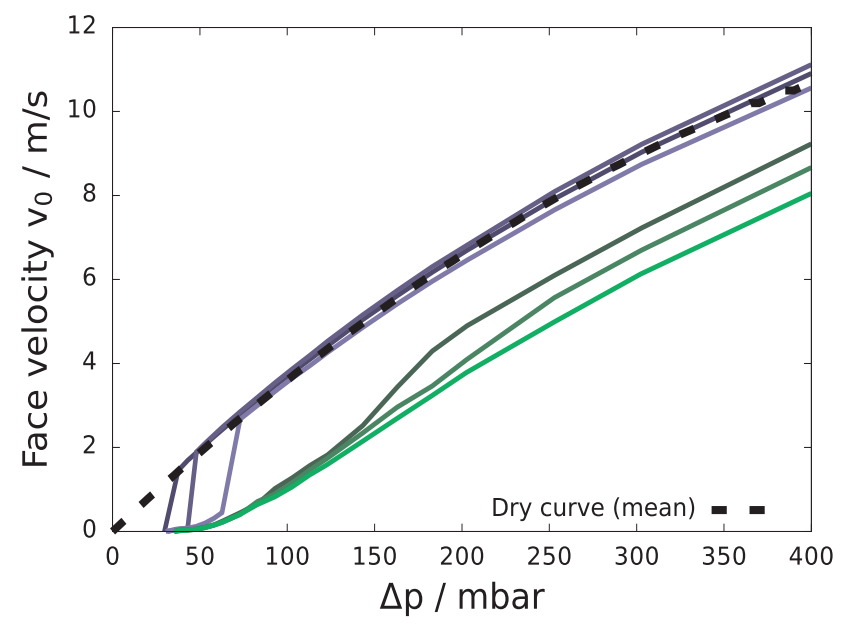

$5 \mathrm{~mm}^{2} / \mathrm{s}$ and $100 \mathrm{~mm}^{2} / \mathrm{s}$. Besides their negligible volatility, silicone oils have the added advantage that their viscosity is variable over a wide range via the chain length of the polymer without much change in surface tension (cf. Table 1).

Fig. 7 shows that the resulting pore size distributions differ very little indeed for such a wide range of viscosities. Largest pores for AK 5 and AK 100 were measured at $21.8 \mu \mathrm{m}$ and $22.5 \mu \mathrm{m}$ and mean pores range from 4.9 to $5.1 \mu \mathrm{m}$. These values do not significantly differ from those of AK 10 given in Table 2.. In fact the differences are hardly visible at the scale of the graphs and certainly much smaller than those caused by volatility effects of some of the working fluids in Fig. 2. We therefore think that the discrepancies observed by Yunoki et al. be tween alcohol, Porofil ${ }^{\mathrm{TM}}$ and ethylene glycol (which lie in a much narrower range of viscosities than our silicone oils) may have also been due mostly to volatility effects.

\subsection{Influence of a variation of scan speed using wetting fluids of different volatility}

Due to the evaporation kinetics of a wetting fluid, the scan time is expected to have a significant and non linear effect on the resulting pore size distribution. This is illustrated in Fig. 8 for the two extremes, namely Porofil $^{\mathrm{TM}}$ and silicone oil (AK 10), by varying scan time incre ments between $1 \mathrm{~min}$ and $120 \mathrm{~min}$. The resulting total scan times thus range from $25 \mathrm{~min}$ to $38 \mathrm{~h}$ !

In case of a volatile liquid such as Porofil ${ }^{\mathrm{TM}}$ (blue curves in Fig. 8), decreasing the scan time shifts the point of rapid evaporation notice ably toward higher pressures. Although the accessible range of pore sizes is thus extended toward smaller pores, a significant cut off cannot be avoided even with very rapid scans. For extremely long scanning times, one may even obtain monodisperse pores in the range of the bubble point. (Another indication that the airflow through the media is a major factor in the evaporation rate.)

For a non volatile wetting fluid such as silicone oil, on the other hand, the effect of scan time on the pore size distribution is compara tively insignificant (green curves in Fig. 8). Only a side by side com parison between the three measurements reveals small differences which are reflected more clearly in the raw data of flowrate vs. $\Delta \mathrm{p}$ (left hand Fig. 8). Between about 150 and $250 \mathrm{mbar}$, the wet curves for scan time intervals of $10 \mathrm{~min}$ and $120 \mathrm{~min}$ each show a small step like in crease, which is more pronounced for the slowest scan rate. We think this may result from a tiny loss of liquid, which is induced by bubbles that form and burst occasionally on the surface of the media, thereby carrying away a few micro droplets. This process, called liquid en trainment is known to occur on the surface of wetted fibrous filters subject to an air flow [16]. In our case it causes a tiny reduction of liquid, which corresponds to a small but noticeable reduction in $\Delta \mathrm{p}$. The step

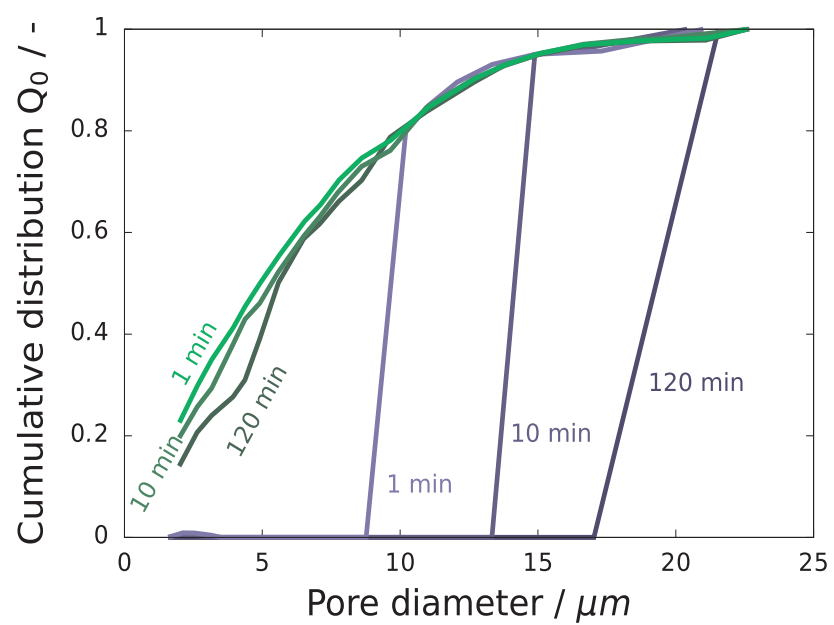

Fig. 8. Effect of scan time interval $(1,10,120 \mathrm{~min})$ on the pore size distribution for Porofil ${ }^{\mathrm{TM}}$ and silicone oil (AK 10). The left-hand figure shows the corresponding wet and dry curves. 


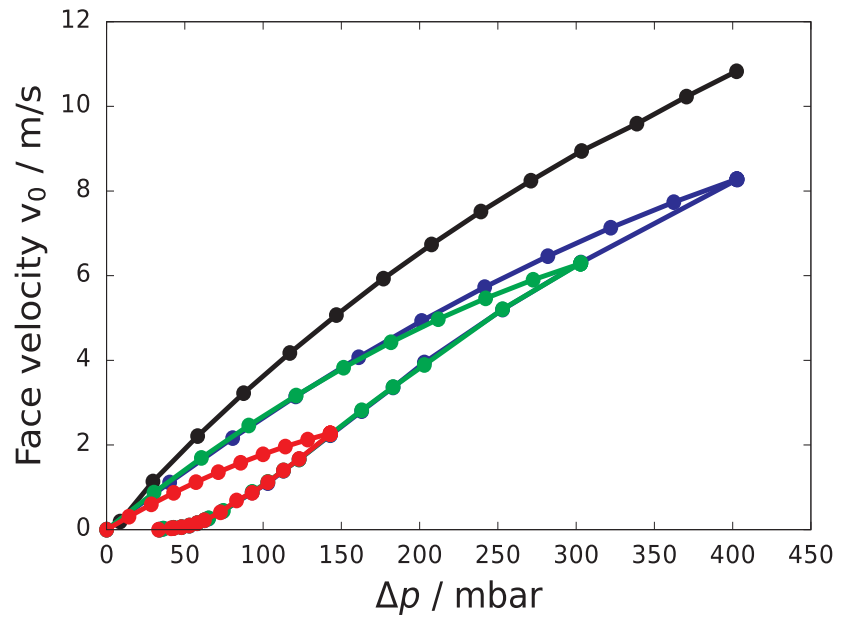

Fig. 9a. "True" dry curve (black) compared wet-followed-by-dry scans, measured for $\Delta$ p endpoints of $150 \mathrm{mbar}$ (red), $300 \mathrm{mbar}$ (green), and $400 \mathrm{mbar}$ (blue). (For interpretation of the references to color in this figure legend, the reader is referred to the web version of this article.)

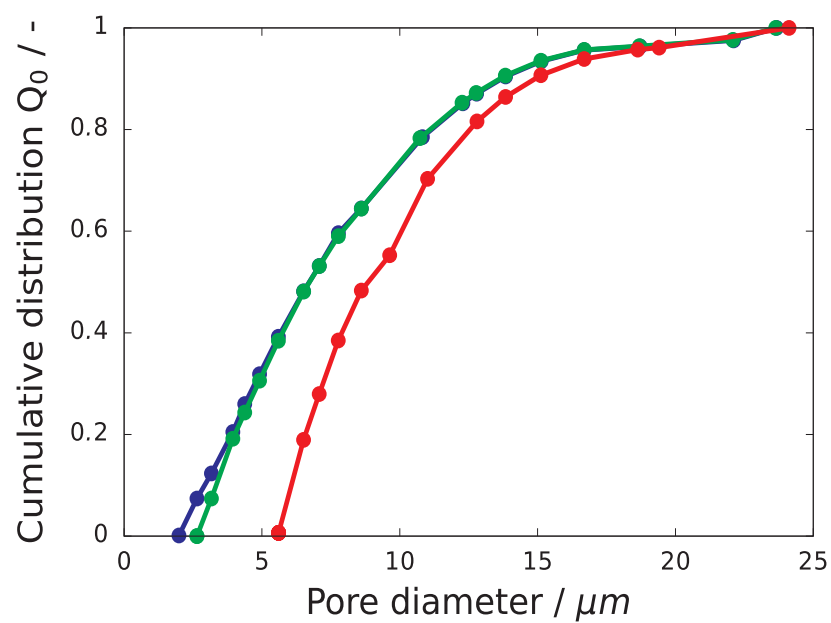

Fig. 9b. Pore size distributions obtained from wet-followed-by-dry scans resulting in mean pore sizes of $6.7 \mu \mathrm{m}$ (green, blue) and $8.9 \mu \mathrm{m}$ (red). (For interpretation of the references to color in this figure legend, the reader is referred to the web version of this article.)

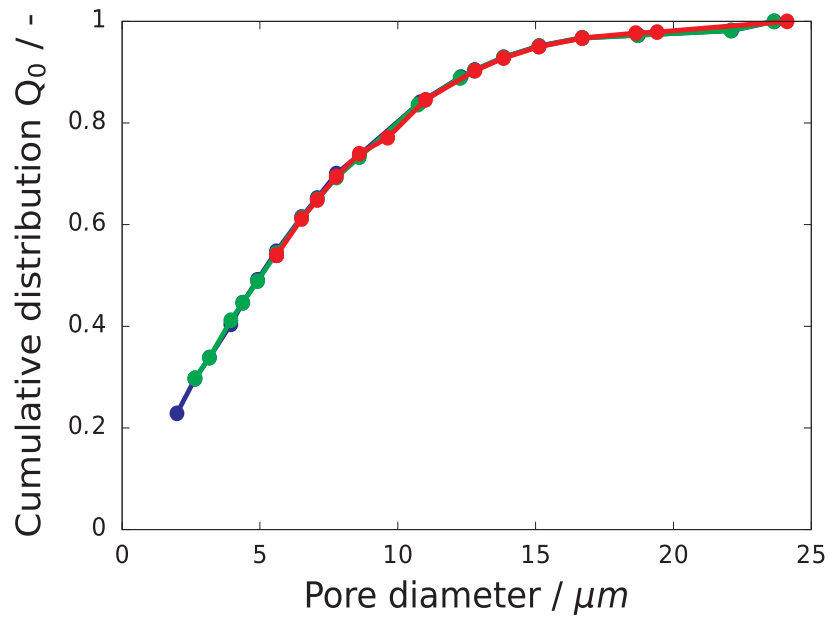

Fig. 9c. Pore size distributions obtained from the "true" dry curve of Fig. 9a resulting in a constant mean pore size of $5 \mu \mathrm{m}$. 
effect appears in a certain $\Delta \mathrm{p}$ range, which marks the transition of foaming and a wet surface and is therefore also most pronounced (i.e. over a narrower $\Delta \mathrm{p}$ range) for the longest scan time interval. It is un derstood however, that such extremely slow scans (total scan time > 1 day) are unrealistic in practice.

\subsection{Influence of the order of the dry and wet scan (only for a non volatile wetting fluid)}

Most commercial porometer software allows a user to choose the order in which the wet and dry measurements are made. Unless otherwise noted, our experiments always start with the "true" dry curve, after which the media are saturated for the wet run. It is indeed tempting to accelerate a pore size distribution measurement by starting with the wet media, blowing them out up to the maximum $\Delta \mathrm{p}$ (which does not necessarily mean completely), and then measuring the "dry" curve on the way down. In the case of a volatile wetting liquid, this may actually work because the liquid has evaporated during the scan. We will not consider volatile liquids any further however, due to the pro blems discussed in the preceding sections. Instead we will concentrate on a non volatile liquid, where this convenient procedure still leads to a systematic error in the resulting pore size distribution, as shown below.

Fig. 9a compares a "true" dry curve (in black) to three "wet before dry" curves in blue, green and red. The difference between the latter is in the $\Delta \mathrm{p}_{\max }$, which was set to 150,300 and 400 mbar, respectively. Note first of all that, of necessity, the upper ends of a pair of curves always meet in cases of a "wet before dry" run. Consequently, the calculated value of $Q$ automatically becomes zero at the end point (the smallest pore). This suggests, erroneously, that one has captured the entire pore size distribution. Secondly, the slopes of these false "dry curves" depend on the chosen $\Delta \mathrm{p}_{\max }$. As a result, the calculated size distributions for different $\Delta \mathrm{p}$ endpoints do not overlap (Fig. $9 \mathrm{~b}$ ), as they should, and as they correctly do when using the "true" dry curve (Fig. 9c).

Instrument manufacturers are apparently aware of this fact. Occasionally, the argument is made nevertheless, that a wet before dry sequence would actually represent the "relevant pore size distribution". Of course, a decision about what part of a pore size distribution is re levant should be made after a measurement, and not beforehand. And it should not be preempted by the measurement itself.

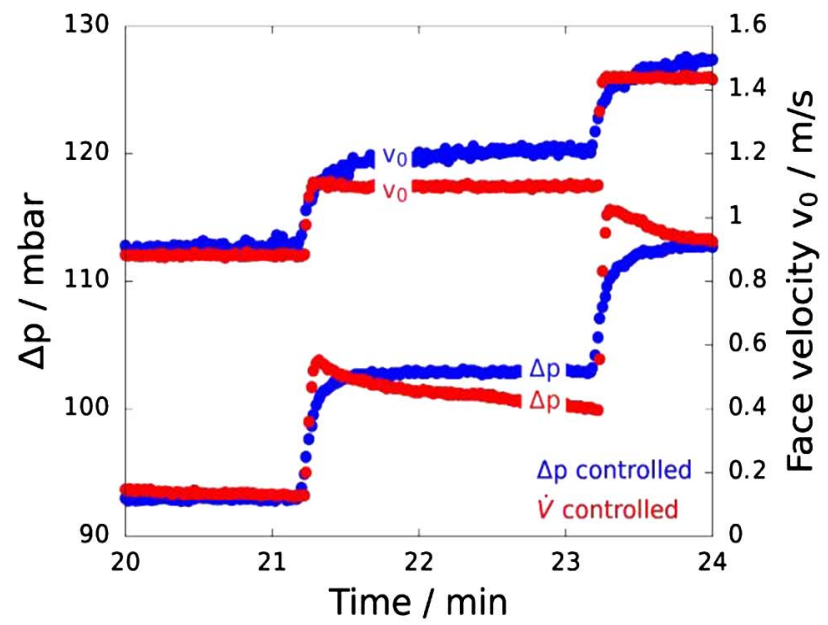

\section{5. $\Delta p$ controlled scan $v s . \dot{V}$ controlled scan}

Porometers are operated either with a pressure controlled or a flow controlled scan sequence. During a scan, the controlling variable is typically changed in small increments at regular time intervals, while the dependent variable "follows". Scan time intervals tend to be as short as possible, in order to speed up the measurement. For that reason, some commercial systems prefer to increase the airflow continually rather than in steps.

Investigations related to the filtration of droplet aerosols and liquid transport of coalesced liquid in fibrous filter media have shown that step changes in airflow rate do not only lead to an opening up of ad ditional pores a fundamental premise of flow porometry but also to an internal rearrangement of the liquid, due to the interconnectivity of pore regions. Depending on the viscosity of the liquid and the structure of the media, the rearrangement can take a considerable amount of time, during which the pressure drop asymptotically approaches its new, stable value $[17,18]$. The influence of scan sequence and scan rate on porometer measurements was therefore addressed.

The left hand graph of Fig. 10 shows representative step changes for both $\dot{V}$ controlled (red) and $\Delta \mathrm{p}$ controlled (blue) measurements and the resulting adjustment of the dependent variable. In each case, the dependent variable takes some time stabilize. In case of a step change in $\dot{V}$ the pressure drop tends to overshoot due to the way mass flow controllers work, while for a step change in $\Delta p$ the volumetric flow rate approaches its new value from below. An overshooting $\Delta \mathrm{p}$ will in principle empty out smaller pores than corresponds to the momentary value of $\dot{V}$, and this effect could shift the pore size distribution towards smaller pores. Fig. 10 shows however, that the effect is not visible in the pore size distribution, at least not for the glass fiber media used for the test, possibly because the open structure of the media permits these smaller pores to be refilled quickly with liquid from neighboring pores or from above the sample.

\section{Summary and conclusions}

Error sources in capillary flow porometry applied to microfibrous media were investigated experimentally. This well established method is based on the gradual displacement of a saturating liquid from inside the media by gradually increasing the differential pressure of an air flow. The pore size distribution is obtained on the basis of classical capillary theory from the corresponding flow rates through the dry and partially "blown out" media.

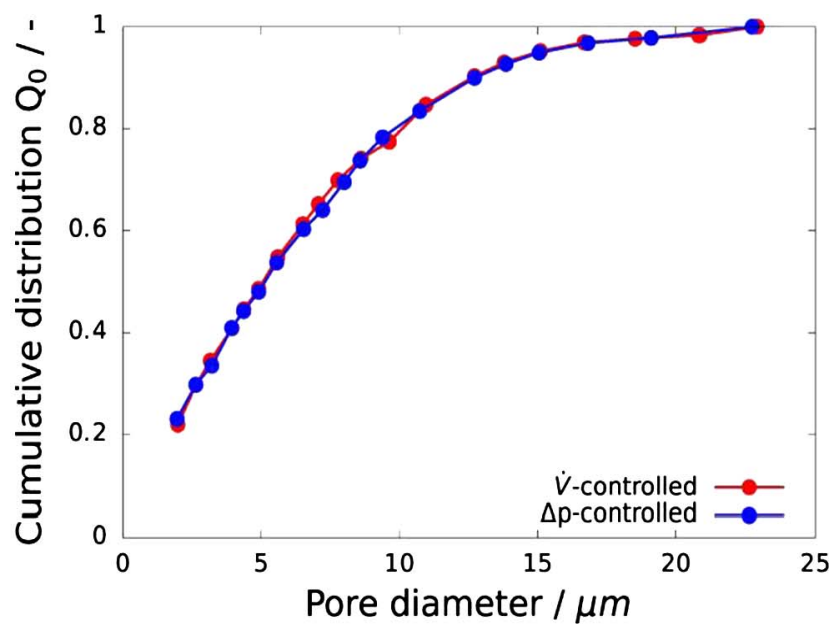

Fig. 10. LEFT: Enlarged sections of raw data for a pressure controlled (blue) and a flow controlled scan (red) using silicone oil AK 10 and a scan time interval of 2 min. RIGHT: pore size distributions corresponding to a pressure controlled (blue) and a flow controlled scan (red). (For interpretation of the references to color in this figure legend, the reader is referred to the web version of this article.) 
Among the factors (i.e. potential error sources) investigated were

- the volatility and viscosity of various wetting liquids,

- the scan speed,

- the order of the wet and dry scans (i.e. dry before wet, or wet before dry),

- $\Delta$ p controlled scans vs. $\dot{V}$ controlled scans.

Experiments were done with a porometer of in house design (cf. Fig. 2), in order to be able to access and control all process variables. Four different wetting fluids were investigated (cf. Table 1). For better comparison, all measurements were made with the identical batch of glass microfiber media.

The by far largest source of errors was due to the volatility of the fluorocarbon wetting fluids used in the study. The vapor pressures of these liquids are generally quite low (exact data are difficult to obtain). Nevertheless, even the slightest air flow through the media beginning with the biggest pores causes these fluorocarbons to evaporate more or less quickly and completely during a scan. The implications for the resulting pore size distribution are enormous, not only with regard to a variation of scan speed and the order of the scans, but also for a several other parameters not investigated in this paper, such as the initial amount of supernatant liquid (which has to evaporate), or ambient pressure and temperature. Evaporation during the measurement of the wet curve results in a complete and premature convergence of the wet and dry curves, and thus in a substantial narrowing of the pore size distributions. Pore sizes below about $5 \mu \mathrm{m}$ are typically not detected. These volatility effects provided a sufficient explanation for differences in pore size data observed between various commercial porometers.

Evaporation effects were not observed in the case of silicone oil based wetting liquids. For these non volatile liquids, the influence of viscosity on the pore size distribution was investigated in a range of $1: 20$. This influence was found to be very small, and the remaining differences were presumably due mainly to a gradual loss of liquid from fine, not yet emptied pores. This loss may be caused by some splattering of liquid drops at higher air flows and therefore seems to depend on the length of the scan time interval. The effect deserves a more detailed investigation in future work. However, intervals of $2 \mathrm{~min}$ had no ap preciable influence on the accuracy.

It was shown that dry before wet sequences deliver consistent pore size distributions that are independent of the maximum $\Delta \mathrm{p}$ of the scan. Furthermore, there was no appreciable influence on the pore size dis tribution, whether a scan was $\Delta \mathrm{p}$ controlled or flowrate controlled.

In final conclusion, volatile wetting liquids may have the advantage of a better wettability for investigations of the largest pores in fibrous media. This small advantage is outweighed however, by the errors in troduced with regard to the almost arbitrary shape of the pore size distribution. The consistent use of non volatile silicone oils is therefore recommended and will make it easier to compare published data.

\section{Acknowledgements}

The authors would like to thank Atlas Copco Airpower n.v. for funding and support of this work as well as Topas GmbH for lending us their equipment for measurements cited in this paper.

\section{References}

[1] F. Erbe, Die Bestimmung der Porenverteilung nach ihrer Größe in Filtern und Ultrafiltern, Kolloid-Zeitschrift 63 (1933) 277-285.

[2] K.R. Morison, A comparison of liquid-liquid porosimetry equations for evaluation of pore size distribution, J. Membr. Sci. 325 (2008) 301-310.

[3] J. Yu, X. Hu, Y. Huang, A modification of the bubble-point method to determine the pore-mouth size distribution of porous materials, Sep. Purif. Technol. 70 (2010) 314-319.

[4] C. Agarwal, A.K. Pandey, S. Das, M.K. Sharma, D. Pattyn, P. Ares, A. Goswami, Neck-size distributions of through-pores in polymer membranes, J. Membr. Sci. 415 (2012) 608-615.

[5] ASTM F316-3, Standard test method for pore size characteristics of membrane filters by bubble point and mean flow pore size, 2003.

[6] A. Hernández, J.I. Calvo, P. Prádanos, F. Tejerina, Pore size distributions in microporous membranes. A critical analysis of the bubble point extended method, J. Membr. Sci. 112 (1996) 1-12.

[7] X.-H. Qin, S.-Y. Wang, Filtration properties of electrospinning nanofibers, Appl. Polym. Sci. 102 (2006) 1285-1290.

[8] D. Li, M.W. Frey, Y.L. Joo, Characterization of nanofibrous membranes with capillary flow porometry, J. Membr. Sci. 286 (2006) 104-114.

[9] J.I. Calvo, A. Hernández, P. Prádanos, L. Martínez, W.R. Bowen, Pore size distributions in microporous membranes II. Bulk characterization of track-etched filters by air porometry and mercury porosimetry, J. Colloid Interface Sci. 176 (1996) 467-478.

[10] S. Madhugiri, B. Sun, P.G. Smirniotis, J.P. Ferraris, K.J. Balkus, Electrospun mesoporous titanium dioxide fibers, Micropor. Mesopor. Mater. 69 (2004) 77-83.

[11] P. Aggarwal, H.D. Tolley, M.L. Lee, Characterizing organic monolithic columns using capillary flow porometry and scanning electron microscopy, Anal. Chem. 84 (2012) 247-254

[12] M.J. Lehmann, E.H. Hardy, J. Meyer, G. Kasper, MRI as a key tool for understanding and modeling the filtration kinetics of fibrous media, Magn. Reson. Imag. 23 (2005) 341-342.

[13] J. Hoferer, M.J. Lehmann, E.H. Hardy, J. Meyer, G. Kasper, Highly resolved determination of structure and particle deposition in fibrous filters by MRI, Chem. Eng. Technol. 29 (2006) 816-819.

[14] T. Yunoki, K. Matsumoto, K. Nakamura, Pore size distribution measurements of nonwoven fibrous filter by differential flow method, Membrane 29 (2004) 227-235.

[15] J.S. Dixon, The effects of wetting fluid vapor pressure on capillary flow porometry, in: Membrane Technology/Separations Planning Conference, 1998, pp. 219-226.

[16] S. Wurster, J. Meyer, G. Kasper, On the relationship of drop entrainment with bubble formation rates in oil mist filters, Sep. Purif. Technol. 179 (2017) 542-549.

[17] T. Frising, D. Thomas, J.C. Appert-Collin, S. Calle-Chazelet, P. Contal, Influence of liquid aerosol stop-and-go on the performance of fibrous filters, Filtration 5 (4) (2005) 286-294.

[18] A. Bredin, B.J. Mullins, Influence of flow-interruption on filter performance during the filtration of liquid aerosols by fibrous filters, Sep. Purif. Technol. 90 (2012) 53-63. 


\section{Repository KITopen}

Dies ist ein Postprint/begutachtetes Manuskript.

Empfohlene Zitierung:

Kolb, H. E.; Schmitt, R.; Dittler, A.; Kasper, G.

On the accuracy of capillary flow porometry for fibrous filter media. 2018. Separation and purification technology, 199.

doi: $10.5445 / / R / 1000080393$

Zitierung der Originalveröffentlichung:

Kolb, H. E.; Schmitt, R.; Dittler, A.; Kasper, G.

On the accuracy of capillary flow porometry for fibrous filter media. 2018. Separation and purification technology, 199, 198-205.

doi:10.1016/j.seppur.2018.01.024 